\title{
A Compound Parabolic Concentrator Coupled to an Optical Microlenslet Array for Projection Display
}

\author{
Heng ZHAOª, Qing YAN, Jun WANG and Deng-Xin HUA
}

\author{
Department of Mechanical and Precision Instrument Engineering, Xi'an University of Technology, 5 \\ South Jinhua Road, Xi'an, Shaanxi, PR China
}

hzhao@xaut.edu.cn

Keywords: Micro-projection display, Compound parabolic concentrator, Microlenslet array.

\begin{abstract}
Regarded as the fourth-generation light sources, light emitting diodes (LEDs) have recently gained much interest as projection light sources due to their long lifetimes, large color gamut and small size. In this work, we design and fabricate a compound parabolic concentrator (CPC) coupled to an optical microlenslet array as a light collection engine of LED-based microprojection diaplay. The results indicate that more than $90 \%$ light can be collected by the CPC coupled to an optical microlenslet array and transmitted within the designed angle. This method is advantageous in many respects compared with those available, such as compact volume, high collection efficiency, rectangular radiation pattern and controllable output divergence angle. This research leads to the idea of possible use of a CPC coupled to a microlenslet array in the domain of micro-projection display.
\end{abstract}

\section{Introduction}

Today, projection displays are used in a variety of configurations, including consumer products, presentation products for the institutional market, large theater displays, very large field-of-view systems for simulator application, and physically small displays for personal use. Regarded as the fourth-generation light sources, light emitting diodes (LEDs) have recently gained much interest as projection light sources due to their long lifetimes, large color gamut and small size. Historically, projectors have served to produce the larger image size while direct-view displays have served to produce the smaller images. The correlation between size and technology appears to be breaking down. Micro-projection technologies can make cost-effective small display that fits in a shirt pocket. So LED-based 'pico-projectors' with typically the size of a cell phone, are beginning to appear in the marketplace. Optical, mechanical and electronic aspects are covered, as they all are tightly intertwined in pico-projectors. Yet miniaturization imposes unique requirements, shift priorities and calls for innovative solutions. There is a bottleneck that the luminous output of LEDs is still lower than that of UHP lamps in micro-projection display. Therefore, it is important to improve light collection efficiency of LEDs for micro-projection display application [1].

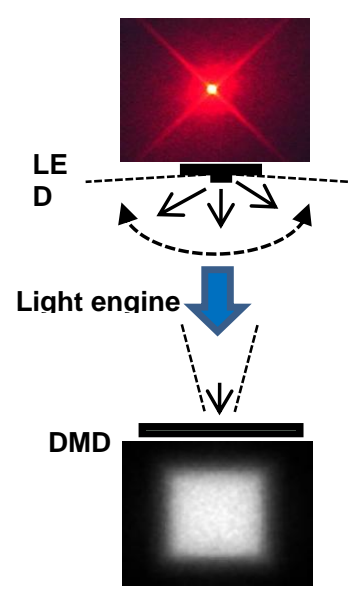

Figure 1. Relationship between LED, light engine and DMD. 
A micro-projector consists of three parts: light source, light engine, projection lens. More important, at the heart of all projectors is the light engine. The design of a projector requires key components to execute the light engine. Traditionally, an LED die is encapsulated in a dome lens. Since the panel of the micro-projector is rectangular and the projection system is an étendue-limited system, the light beam emitting from the LED should be collimated and homogenized by the light engine, which transforms the circular radiation pattern to rectangular radiation pattern within a certain acceptance angle. Optical improvements allow more of the light extracted from the die to be focused on the digital micro-mirror device (DMD), as shown in Fig. 1. However, the challenge is how to collect as much light as possible from a large, wide-angle LED, to direct it on a DMD and then to enter the projection lens.

In this work, a compound parabolic concentrator (CPC) coupled to an optical microlenslet array is designed and fabricated as a light collection engine of LED-based micro-projection display. One of the great advantages of this configuration is its great light collection efficiency. There is no auxiliary focusing lens required in this configuration, another advantage which simplifies the overall system architecture [2].

\section{Optical Design and Simulation}

\section{Modeling of a CPC}

In this work, a $3 \times 4$ monolithic LED chip is encapsulated in the bottom recess of the CPC as a micro-projection source, as shown in Fig. 2. The incident angle of rays is required as about $12^{\circ}$ for the DMD device of microdisplay.
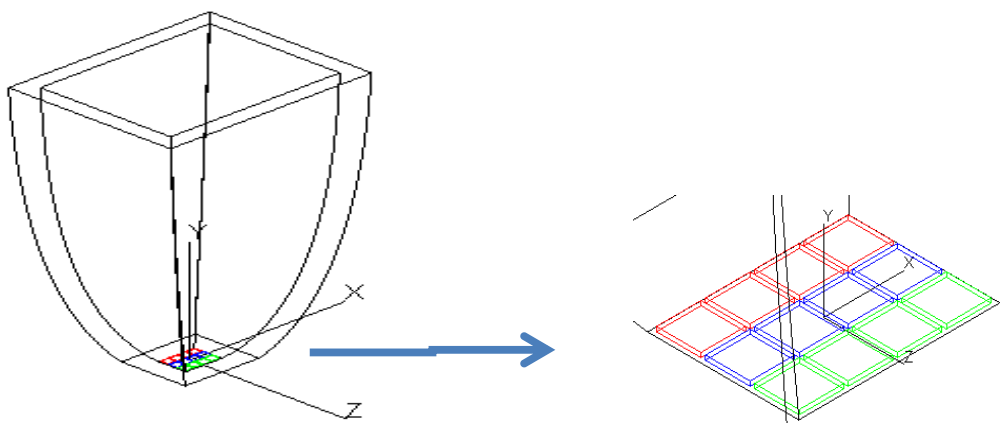

Figure 2. Schematic of $3 \times 4$ monolithic LED chip.

Based on the theory of étendue from non-imaging optics and the edge ray principle, in combination with the theory of freeform surfaces, this paper involves mathematical models of the CPC freeform surfaces, which are solved by numerical method to obtain the shape of surfaces with 3D modeling software ProE®.

A proper design of a rectangular CPC is given. The max angle $\theta_{i}$, with which light rays reach the exit aperture of the CPC is given by

$$
\operatorname{Sin} \theta_{\max }=a^{\prime} / a
$$

where $\mathrm{a}^{\prime}$ is the semi-diameter of the entrance aperture, and a is the semi-diameter of the exit aperture, as shown in Fig. 3. Equations should be centred and numbered with the number on the right-hand side.

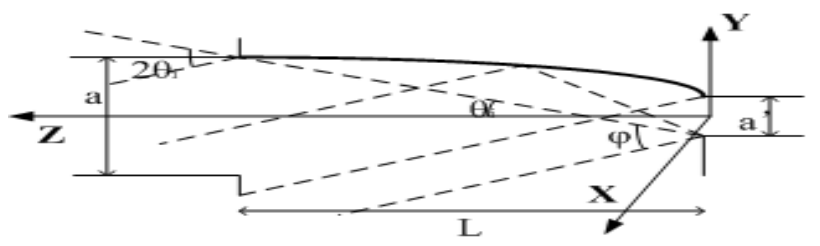

Figure 3. Schematic of CPC. 
The focal length of the parabolic curve is

$$
f=a^{\prime} /\left(1+\sin \theta_{i}\right)
$$

The length of the CPC is given by

$$
L=\left(a^{\prime}+a\right) / \tan \theta_{i}
$$

\section{Modeling of an Optical Microlenslet Array}

A practical approximation for the irradiance distribution over the microlenslet array is given by Eq. 4.

$$
E(r, \theta)=E_{0}(r) \cos ^{m} \theta
$$

where $\theta$ is the viewing angle and $\mathrm{E}$ is the irradiance on axis at distance $\mathrm{r}$ from the LED array. The value of $m$ depends on the relative position of the LED chip emitting region from the curvature center of the parabolic curve of CPC [3].

The irradiance distribution given by Eq. 5 for a LED displaced to position ( $\left.\mathrm{x}_{0}, \mathrm{y}_{0}\right)$ over the microlenslet array can be written in terms of Cartesian coordinates $(\mathrm{x}, \mathrm{y}, \mathrm{z})$. The irradiance at a point $\mathrm{P}(\mathrm{x}, \mathrm{y}, \mathrm{z})$ then is expressed as

$$
E(x, y, z)=\frac{z^{m} L A}{\left[\left(x-x_{0}\right)^{2}+\left(y-y_{0}\right)^{2}+z^{2}\right]^{(m+2) / 2}}
$$

where $L$ is the radiance of the LED source and $A$ is the area of the microlenslet array.

In structured lighting, the most popular array consists of linear-array lenslets. Consider a linear array with a lenslet-to-lenslet separation $d$. In this case, irradiance $E$ is given by the sum of the irradiances for $\mathrm{N}$ lenslets, as shown in Fig. 4:

$$
E(x, y, z)=z^{m} A L \sum_{n=1}^{N}\left\{[x-(N+1-2 n)(d / 2)]^{2}+y^{2}+z^{2}\right\}^{-(m+2) / 2}
$$

where $\mathrm{L}$ is the radiance of LED source and $\mathrm{A}$ is the area of microlenslet array.

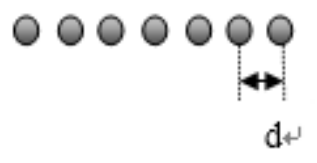

Figure 4. Schematic of linear microlenslet array with separation d.

\section{A Rectangular CPC Coupled to an Optical Microlenslet Array}

A light collection engine consists of several parts: a monolithic LED array, a rectangular CPC and an optical microlenslet array [4]. The Polymethylmethacrylate (PMMA) is used as the material of this configuration.

First, the illumination distribution of the monolithic LED array is simulated by TracePro®. Fig. 5 shows light output pattern of the monolithic LED chip. An illumination-uniformity study can be performed using a radiometric analysis by considering each LED on a monolithic LED chip as a non-perfect Lambertian and incoherent emitter. In practice, this dependence turns out to be a power law that mostly depends on the encapsulant and semiconductor region shapes. 

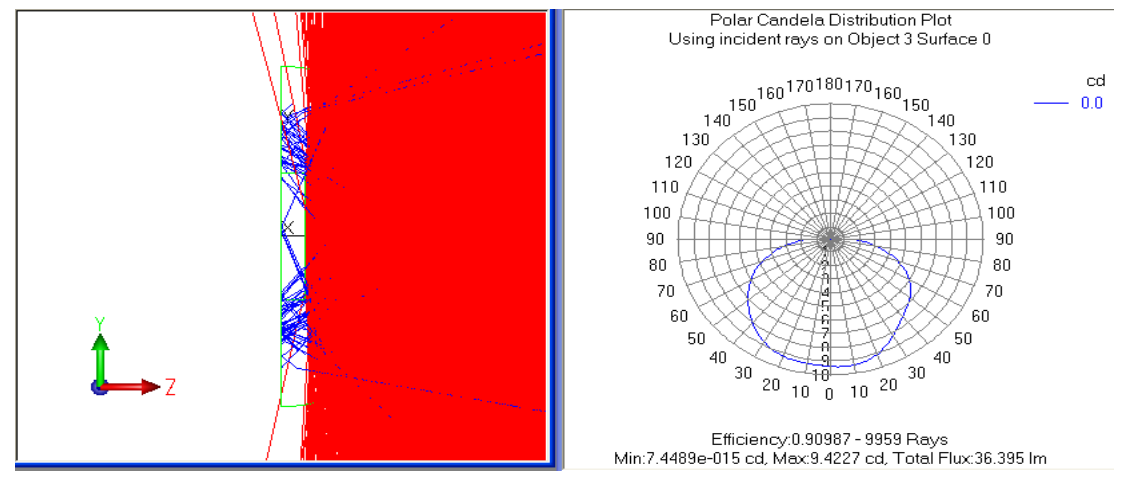

Figure 5. Light output pattern of the monolithic LED chip. (a) Raytrace result. (b) Polar candela light distribution.

The profile of the light collection engine by ProE® is shown in Fig. 6(a). The bottom recess of the rectangular CPC is in the shape of cuboid with a length of $3.2 \mathrm{~mm}$, a width of $2.5 \mathrm{~mm}$ and a height of $0.5 \mathrm{~mm}$. LED dies are encapsulated by the silicone in the recess. From the N.A. of the micro-projector lens, the maximum value of $\theta$ is $12^{\circ}$. By Eq. 3, the length of the CPC is given as $33.7 \mathrm{~mm}$.

Next, the optical lenslet array is coupled together in the top of the CPC. The aperture and focal length of microlenslet are determined according to the size of rectangular CPC and the aperture angle of lighting systems. The diameter of the output aperture is $19.11 \mathrm{~mm}$ and the length of the CPC is $53.16 \mathrm{~mm}$. Upon making use of Eq. 5-6, a $125 \times 125$ micro array is obtained as the structure of lenslet array with $80 \mu \mathrm{m}$ in diameter.
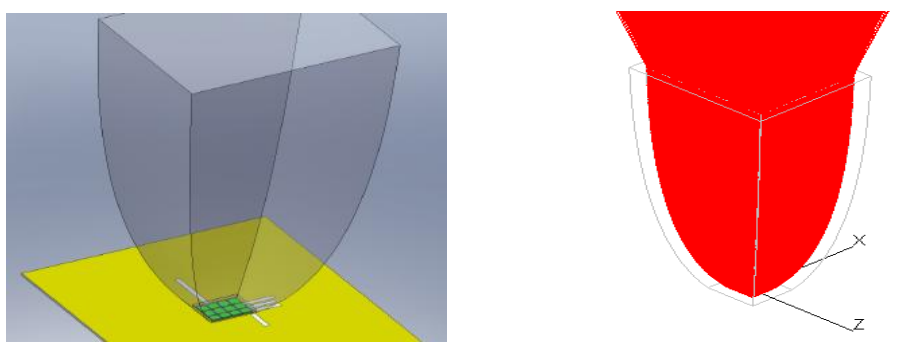

Figure 6. Layout of CPC coupled to optical lenslet array. (a) 3D view. (b) Front view with light rays.

All components of the optical system are held inside a PMMA cylinder to make the system mechanically feasible. The monolithic LED array is put at the bottom of the CPC with a distance of $0.1 \mathrm{~mm}$, and the entrance area of the CPC covers the LED array completely. Meanwhile, the optical lenslet array is set at the top of the CPC, as shown in Fig. 6(a). Eventually, the ray tracing results are shown in Fig. 6(b).

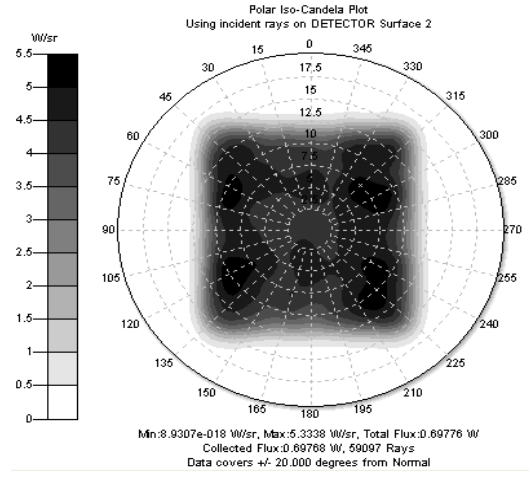

(a)

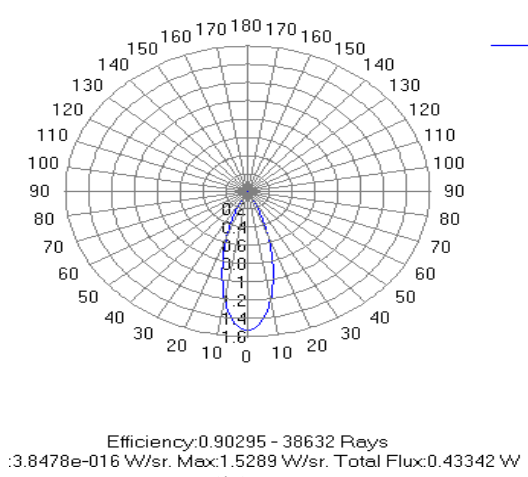

(b)

Figure 7. Simulated result. (a) Uniformity of exit light intensity distribution for light collection engine. (b) Polar candela distribution for light collection engine. 
Simulation results are shown in Fig. 7. Fig. 7(a) pictures the simulated uniformity of the light collection engine. It is shown that the light collection engine has a high collection efficiency of 90.3\%.The uniformity is also an important parameter in projection display application. Furthermore, variations of the exit light intensity across the target surface are typically minor based on the coupled structural design. In addition, it should be noted that the light collection engine has the capacity where the intensity is uniform across distribution angle from $-12^{\circ}$ to $12^{\circ}$. Then, the intensity gradually falls off, as shown in Fig. 7(b). This result shows that the system can satisfy the design requirement.

\section{Experimental Results}

In this paragraph the experimental setup of the designed the light collection engine is made, along with experimental the results. A comparison of simulated model and the experimental setup is then given. After that, the performances of the demonstrator are measured and the results are discussed. Finally, some suggestions are presented to improve the light engine in the near future.

According to the simulation results, the light collection engine is fabricated as followed. The production process of the optical lenslet array is first briefly introduced [5].

Step 1: Acid-assisted rapid manufacture of the lenslet mold. The glass mold of the array with rectangular shape is prepared in this section. First, a femtosecond laser beam is carried out point-bypoint on a polished silica glass chip $\left(10 \times 10 \mathrm{~mm}^{2}\right)$ with a 30 -fs and $800-\mathrm{nm}$ laser pulses at a repetition of $1 \mathrm{kHz}$. Second, the sample with craters is treated in hydrofluoric acid solution assisted by an ultrasonic bath at about $23^{\circ} \mathrm{C}$. During this process, the chemical etch is significantly enhanced in the laser-induced craters, and the concave spherical surfaces begin to form in the laser-exposure spots [6]. The circular-shaped concave structures expand with the chemical etching process. When the neighbor ones contact with each other, straight boundary lines are formed, eventually forming the hexagonal patterns, as shown in Fig. 8(a).

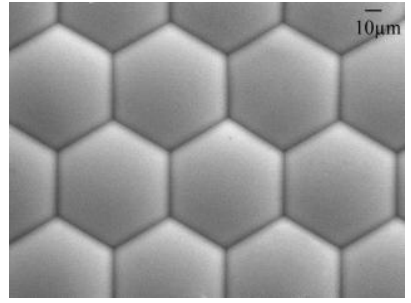

(a)

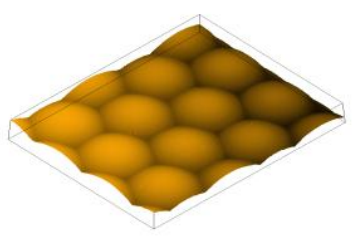

(b)

Figure 8. Actual sample of optical lenslet array. (a) SEM image of optical lenslet array. (b) 3D profile of array which is captured by 3D laser confocal microscope.

Step 2: Inject molding. For replication of micro-structures, the PMMA sheet and mold were fixed onto upper and lower stages of the system [7,8]. The glass mold is heated to $120^{\circ} \mathrm{C}$ by an electric heating template with a power of $400 \mathrm{~W}$. The PMMA sheet is moved downwards and pressed on the heated mold. As the heating process terminates, the temperature declines gradually. At the temperature below $50^{\circ} \mathrm{C}$, the process of stripping PMMA sheet is made to form the microlenslet array. Figs. 8(b) shows the SEM images of the mold, a fact which demonstrates that the uniform and smooth surface can be made by this method.

Step 3: Bond molding. According to the design of CPC earlier, the dielectric rectangular CPC is fabricated with PMMA by injection molding, extrusion and casting processing. Then, it bonds well with the microlenslet array previously prepared by the thermal pressed treatment [9]. Finally, the light collection engine comes into shape with the structure of the dielectric rectangular CPC coupled to an optical microlenslet array, as shown in Fig. 9(a).

The performance of red LED module is then tested and characterized. There is shown that a radiation pattern of integrated with red LED modules is under power-on test in Fig. 9(b). It is found that the output radiation pattern has been transformed to the rectangular pattern which is matched 
well with the microdisplay panel. It is noted that the light beam emitting from a monolithic LED array has been collimated effectively. The divergence angle of the light beam exiting the multipleLED module is $13^{\circ}$, which is consistent with the computer emulation.

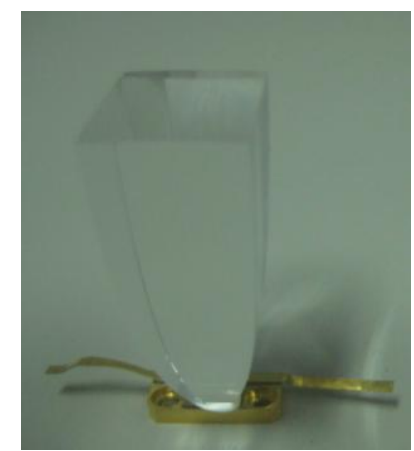

(a)

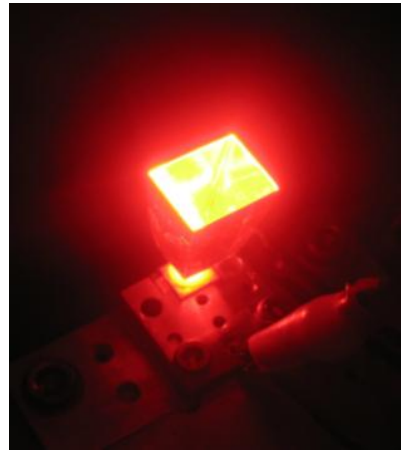

(b)

Figure 9. Prototype test on the light collection engine packaged a red LED module. (a) The photograph of the actual sample. (b) The picture of the light patterns for the demo.

The uniformity calculation of the image is specified by an ANSI/NAPM IT7.228-1997 (ANSI/ NAPM, 1997) named 13-point measurement, which has been widely employed in display measurements. As shown in Fig.10, thirteen sample points are distributed over the measurement area. Conventionally, average illuminance is obtained by computing the average illuminance value of nine points, and uniformity is the ratio of minimum illuminance among thirteen sample points to the average illuminance, as shown in Eq. 7.

$$
\text { Uniformity }=\frac{\min (1 \sim 13)}{\text { average }(1 \sim 9)} \times 100 \%
$$

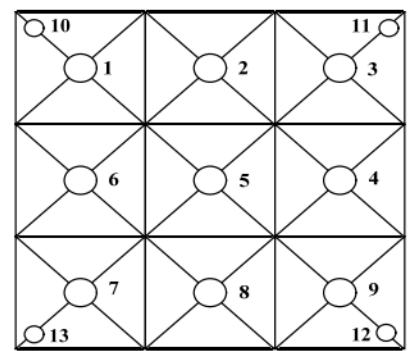

Figure 10. Schematic of 13-point measurement.

As a result, the uniformity of the image on screen is calculated to be $82.7 \%$. The result obtained demonstrates that the uniformity is slightly lower than the simulation one with $90.3 \%$. Many reasons cause the difference, including fabrication error, the size of device, the intensity distribution of LED light source, and so on. Moreover, the simulation result is done based on the ideal situation that the intensity distribution of the LED is uniform and the angle distribution is Lambertian. Actually, there is a little difference between the factual status with the ideal condition. Also, the experiment result is greatly affected by the fabrication error. Therefore, there are at least two possible ways to advance the performance of the design. The first way to tackle it is to amend the prototype by the actual measurement data of the intensity/light distribution of an actual LED light source. Another that is worth adopting is to improve the mechanical precision.

\section{Summary}

In summary, a coupled structure is presented for a light collection engine in this paper. A rectangular dielectric CPC coupled to an optical microlenslet array is designed and optimized to improve the light utilization efficiency for micro-projector applications. More than $90 \%$ light 
emitted by a monolithic LED array can be collected by this CPC and transmitted within the designed angle. This method is advantageous in many respects compared with those available, such as compact volume, high collection efficiency, rectangular radiation pattern and controllable output divergence angle. Therefore, this research leads to the idea of possible use of a CPC coupled to a microlenslet array in the domain of micro-projection display.

Moreover, the microstructures with an optical microlenslet array are created in one mold in this work, and the shapes are simply controlled individually by rapid prototyping technology based on femtosecond laser pulses. This method provides an alternating approach for the fast fabrication of large-area polymeric micro-optical devices with various shapes in further study.

\section{Acknowledgement}

The authors would like to acknowledge support from the Scientific Research Programs of Shaanxi Provincial Department of Education grant 12JK0660, as well as the Natural Science Foundation of Shaanxi Provincial Department of Science and Technology grant 2013JQ7002.

\section{References}

1. Y. H. Ra, R. Wang, S. Y. Woo, Nano Lett. 16 (2016)

2. M. Gajic, N. Karwa, A. Mojiri, Opt. Express. 23 (2015)

3. J. Kwapien, S. Drozdz, Phys. Rep. 515 (2012)

4. M. Willizm, G. M. Schuster, J. E. Ford, Opt. Express. 22 (2014)

5. K. H. Jeong, J. Kim, L. P. Lee, Science, 312 (2006)

6. C. C. Huang, X. Wu, H. Liu, Small. 10 (2014)

7. R. Intartaglia, S. Beke, M. Moretti, Lab Chip. 15 (2015)

8. S. Schwarz, S. Rung, R. Hellmannf, . Phys. Lett. 108 (2016)

9. K. Sugioka, J. Xu, D. Wu. Lab Chip. 14 (2014) 